\title{
BMJ Open Quality Optimising the mandatory reporting process for drivers admitted to an inpatient stroke rehabilitation unit
}

\author{
Shannon L MacDonald, ${ }^{1}$ Pamela L Joseph, ${ }^{1}$ Ida J Cavaliere, ${ }^{1}$ \\ Mark Theodore Bayley, ${ }^{1,2}$ Alexander Lo ${ }^{1,2,3}$
}

To cite: MacDonald SL, Joseph PL, Cavaliere IJ, et al. Optimising the mandatory reporting process for drivers admitted to an inpatient stroke rehabilitation unit.BMJ Open Quality 2018;7:e000203. doi:10.1136/ bmjoq-2017-000203

Received 9 September 2017 Revised 19 May 2018 Accepted 15 June 2018
Check for updates

(C) Author(s) (or their employer(s)) 2018. Re-use permitted under CC BY-NC. No commercial re-use. See rights and permissions. Published by BMJ.

${ }^{1}$ Division of Physical Medicine and Rehabilitation, Department of Medicine, University of Toronto, Toronto, Ontario, Canada

${ }^{2}$ Brain and Spinal Cord Rehab Program, University Health Network, Toronto Rehabilitation Institute, Toronto, Ontario,

Canada

${ }^{3}$ University of Toronto Centre for Quality Improvement and Patient Safety, Toronto, Ontario, Canada

Correspondence to

Dr Alexander Lo;

Alexander.Lo@uhn.ca

\section{ABSTRACT}

Ontario physicians are legally obligated to report patients who may be medically unfit to drive to the Ministry of Transportation of Ontario (MTO). Currently at Toronto Rehabilitation Institute (TRI), there are no standardised processes for MTO reporting, resulting in inconsistent communication regarding driving with patients and between healthcare providers, redundant assessments and ultimately reduced patient satisfaction. TRI received 10 patient complaints regarding the driving reporting process in the 5 years prior to this project and a large number of patients were not being reported appropriately. The project aim was to use Lean Methods to achieve $100 \%$ reporting and optimise communication and education of drivers admitted to a 23-bed inpatient stroke rehabilitation unit. Interventions included process mapping, identification of wasteful steps and implementation of a standard work. Chart audits before and after implementation were performed.

Value stream process mapping identified inconsistent reporting procedures and lack of use of the governmentissued driver reporting form. Following implementation of standard work processes, use of the MTO Medical Conditions Report Form increased from $0 \%$ to $100 \%$. Indication of whether drivers were reported to the MTO in Physical Medicine \& Rehabilitation consultation notes increased from $50 \%$ to $91 \%$. Identifying reported drivers in the discharge summary, of which patients receive a copy at the time of discharge, increased from $0 \%$ to $90 \%$. Physician satisfaction with the new standard work process was qualitatively assessed to be high, with no negative impacts reported.

Lean methodology was effective for increasing the usage of the MTO Medical Conditions Report Form, documenting driver status in the initial Physical Medicine \& Rehabilitation consultation and indicating MTO reporting status in the discharge summary.

Communication between healthcare providers regarding patients' driving status has been successfully standardised, resulting in improved coordination of care and a reduction in patient complaints to zero in the 14 months since implementation.

\section{PROBLEM}

Physicians working in Ontario, Canada are legally obligated to report patients who may be medically unfit to drive to the Ministry of Transportation of Ontario (MTO). This law is particularly relevant to our organisation, University Health Network - Toronto Rehabilitation Institute (TRI), which is one of the leading rehabilitation centres in North America. ${ }^{1}$ TRI consists of five stand-alone facilities located in a large urban Canadian city and offers both inpatient and outpatient rehabilitation services. There are approximately 530 inpatient admissions to the stroke and acquired brain injury (ABI) rehabilitation programs per year and a large proportion of these patients have cognitive or physical impairments that may affect driving safety.

To date, there have been no standardised reporting or return to driving processes at TRI. Over the past 5 years, the hospital has received 10 formal patient complaints related to license suspension and lack of information provided regarding the reporting and return to driving process. As a result, we performed an institutional review of these practices across the three inpatient and six outpatient stroke and ABI rehabilitation programmes to identify potential areas for improvement.

Our organisation chose to pilot the project on the Stroke inpatient rehabilitation unit, which is a 23-bed ward. Patients are admitted under a hospitalist physician with consultation services provided by one of two Physical Medicine and Rehabilitation (PMR) specialists. After each patient's initial consultation, the PMR specialist should evaluate whether the patient must be reported to the MTO based on the presence and severity of any physical or cognitive poststroke impairments, and informs the patient regarding their decision. The PMR consultant's note, the hospitalist's admission note and the discharge summary are sent to the community family physician and should include information about the patient's driving status. After discharge from the inpatient stroke rehabilitation unit, many patients continue to be followed at TRI in an interdisciplinary outpatient stroke rehabilitation programme, where return to driving 
is commonly addressed as an outpatient rehabilitation goal. The problem was that the processes above were not consistently followed.

The project aim was to use Lean Methods to achieve $100 \%$ reporting using the official government-issued driver reporting form while increasing the percentage of PMR consultation notes and discharge summaries that document driver reporting status to $>90 \%$ within 12 months. A secondary aim was to optimise communication and education of drivers admitted to a 23-bed inpatient stroke rehabilitation unit.

\section{BACKGROUND}

In 2013, approximately 405000 Canadians living in the community experienced the effects of a stroke, with a prevalence of $1.15 \% .^{2}$ Stroke is a leading cause of disability and death in Canada, costing an estimated $\$ 3.6$ billion in healthcare expenditures and productivity loss. ${ }^{3}$

Stroke can lead to physical and cognitive impairments, including hemiparesis, ataxia, inattention, neglect, reduced processing speed and visuospatial impairments, which can negatively impact driving safety. ${ }^{4}$ In fact, individuals post-stroke have a twofold higher risk of motor vehicle collisions compared with those without stroke. ${ }^{5}$ As a result, identification of high-risk patients post-stroke is crucial for patient and public safety on the road.

National physician guidelines are available regarding medical conditions that may pose a risk to driving safety. ${ }^{4}$ However, there are no available guidelines regarding the optimal process for reporting patients or the appropriate information to provide stroke survivors regarding driving after a stroke. A previous systematic review by Frith found that only half of stroke survivors in acute care were provided with driving education prior to discharge home. ${ }^{6}$ Qualitative studies suggest patients are given limited recommendations and inadequate information about driving poststroke from both acute care and rehabilitation facilities. $^{7}$

Driving is considered an instrumental activity of daily living and the inability to drive negatively impacts patients' ability to reintegrate into the community and participate in work, social and recreational activities. ${ }^{8}$ Qualitative studies have shown that loss of driving privileges negatively impacts patient quality of life and independence. ${ }^{7}$

A systematic process for reporting patients to the MTO would potentially improve consistency in information provided to patients and members of their healthcare team, as well as patient satisfaction.

\section{MEASUREMENT}

A baseline chart audit of patients admitted to the stroke inpatient rehabilitation unit was performed in February 2016. Only 50\% of PMR consultations described a plan to address driving, and the standardised government-issued medical conditions report form was used for none $(0 \%)$ of the patients reported to the MTO. Instead, a triplicate form generated by our institution was sent for all reported patients. We performed a retrospective chart audit of patients discharged in February 2016 and found that none $(0 \%)$ of the discharge summaries stated whether the patient was reported to the MTO.

The selected process measures for our intervention included: (i) percentage of charts using the standardised MTO medical conditions report form, (ii) percentage of patients with driver status documented in the PMR consultation note and (iii) percentage of drivers with MTO reporting status documented in the hospitalist's discharge summary. These process measures were objective, easy to measure and represented important communication methods between the hospitalist, PMR consultant, community family physician and patient. The PMR consultation notes and the discharge summaries are frequently used to communicate with the patient's outpatient rehabilitation team. Furthermore, patients receive a copy of their discharge summary when leaving the hospital, which informs them whether they were reported to the MTO or not. Patients who were not drivers prior to their stroke were excluded from the chart audit. Staff satisfaction was assessed qualitatively as a balancing measure.

Monthly chart audits were performed and the results were plotted and analysed on run charts. The dates of meetings with key stakeholders and staff changeover were indicated on the run charts to contextualise the findings.

\section{DESIGN}

Prior to the intervention, individual physician interviews were performed with nine hospitalists and PMR consultants, which confirmed variability in reporting practices as well as inconsistent communication regarding driving with patients and among healthcare providers.

Process mapping, also known as value stream mapping, was completed in a meeting that included relevant stakeholders, including the Medical Director of the Brain Programme, Medical Doctors, Occupational Therapists, Psychologist and Lean process improvement coaches. Several key opportunities for improvement on the stroke rehabilitation unit were identified: (i) the standard MTO reporting form was not being used, (ii) reporting practices were inconsistent and (iii) there was a lack of communication regarding the MTO reporting decision to patients, community physicians and outpatient teams.

In order to standardise and improve the MTO reporting process, we prioritised steps that would have most impact and aimed to: (i) standardise the use of the MTO's medical conditions report form, (ii) improve consistency in indicating driver status in PMR consultations and (iii) identify reported drivers in the discharge summary.

With support from the Lean process improvement coaches, the process map was used to generate a standard work form outlining the key steps to assess and, potentially, report stroke rehabilitation inpatients' fitness to drive. The major steps, in brief, included the following: (i) a blank MTO medical conditions report form is inserted in the chart on chart assembly; (ii) PMR performs their 
consultation; and (iii) the patient is categorised as unfit to drive, safe to drive or unclear. There are three separate pathways based on this initial categorisation. If the patient is to be reported, the following steps are taken: (i) the MTO form is completed, (ii) patient education is provided, (iii) form is faxed, (iv) faxed copy with confirmation is placed in the chart, (v) the decision to report is documented in the PMR consultation note and (vi) the reporting status is documented in the discharge summary. To ensure staff accountability for the standard work, clear role responsibility for completion of each task was assigned to one team member.

\section{STRATEGY}

We employed iterative quality improvement cycles while collecting data continuously over time. The charts and electronic medical records of patients admitted between February 2016 and April 2017 were audited. Chart audits of PMR consultations and discharge summaries were completed on a monthly basis from February to July 2016, with a retrospective chart audit completed in May and June 2017.

\section{Cycle 1: forcing function}

An education session was held with the hospitalist and PMR consultants to orient them to the new standard work form and address any questions and concerns. With the aid of the ward clerk, a forcing function strategy was employed by removing the previously used triplicate form from the unit to improve usage of the standardised MTO Medical Conditions Report Form. The ward clerk agreed to insert a blank copy of the standard MTO reporting form in a consistent location in the patient chart at the time of admission.

\section{Cycle 2: trainee orientation}

The documented plan for reporting a patient to the MTO was initially inconsistent in the PMR consultation note, increasing to $90 \%$ after 4 weeks, but then dropping to $57 \%$ by 12 weeks. In May 2016, we emailed the PMR specialists to ensure a process was in place to orient trainees (residents and medical students) on service regarding the standard work, as trainees dictate most consultation notes. We confirmed with the PMR specialists that trainees are given specific instructions to include in their consult notes whether patients were reported to the MTO.

\section{Cycle 3: consensus building with hospitalist and further standardising processes}

The communication of MTO status in discharge summaries immediately improved after implementation of the standard work. In June 2016, 60\% of discharge summaries included the MTO status. To further improve results, we had a meeting with the hospitalist to troubleshoot any barriers to including the MTO status in discharge summaries. He had several reference points to identify whether a patient was reported to the MTO, including the PMR consultation notes, written orders in the chart to fax the MTO letter and presence of completed MTO forms on the chart. To facilitate the process, we liaised with other stakeholders, including PMR consultants and the ward clerk, to ensure that an order was written to fax the MTO form and that the MTO form was being placed consistently in the back of the chart for quicker reference.

Cycle 4: e-discharge with computerised automated reminder A new method of generating electronic discharge summaries (e-discharge) was implemented in December 2016 at TRI. Our unit hospitalist was transitioned to typing discharge summaries instead of verbal dictations. The e-discharge included an automated function to indicate whether patients were reported to the MTO. A standard paragraph was included for patient reference, which stated that the MTO was notified, the patient should receive notification from the MTO regarding the process of license re-instatement, and that the patient should not drive until notified by the MTO.

Cycle 5: spread to other units

In February 2017, a Brain Programme physicians' meeting was held. The results of our chart audits were shared.

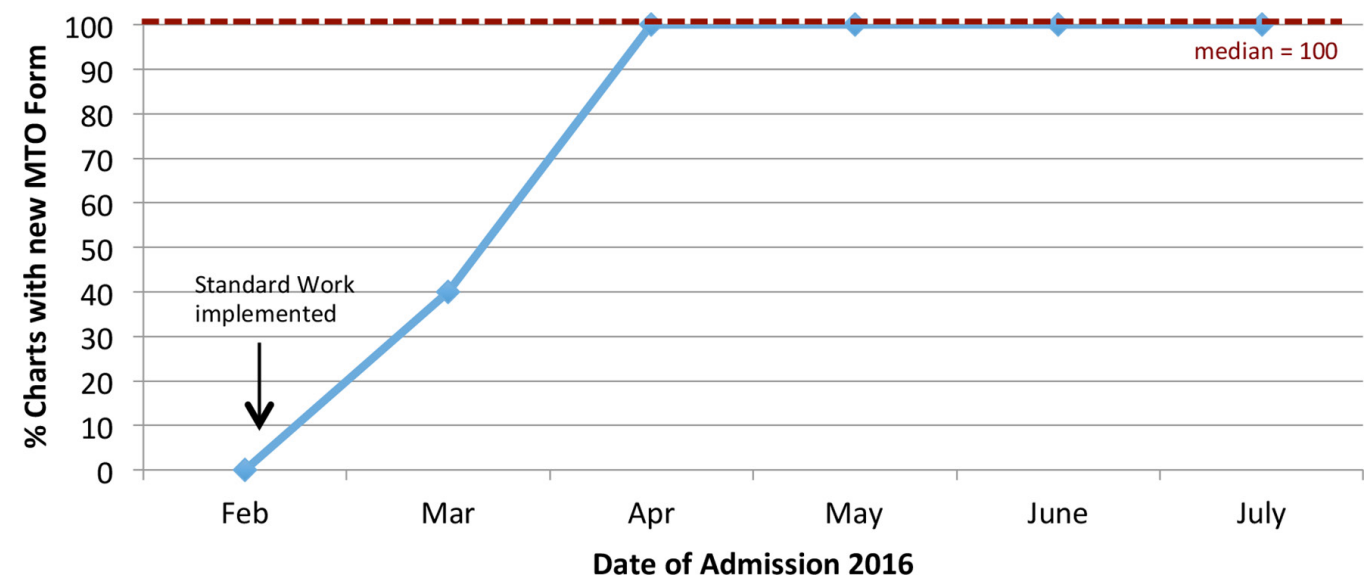

Figure 1 Percentage of charts using the standardised MTO medical conditions report form. MTO, Ministry of Transportation of Ontario. 


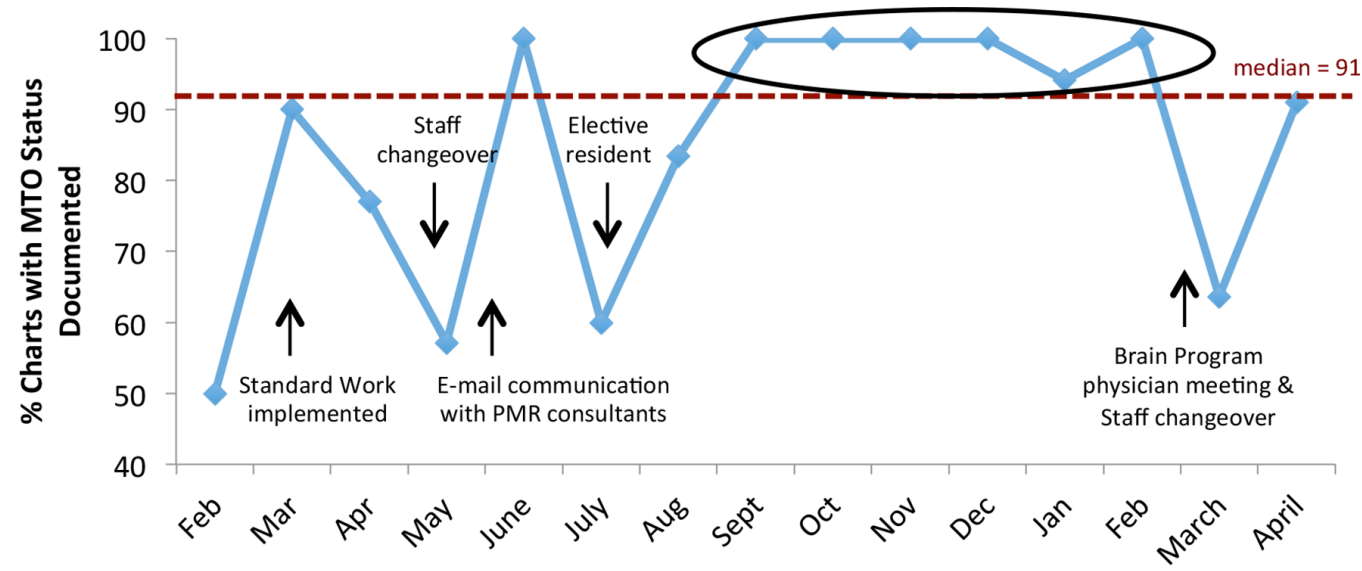

Date of Admission 2016-2017

Figure 2 Percentage of PMR consultations documenting the plan for MTO reporting. MTO, Ministry of Transportation of Ontario; PMR, Physical Medicine and Rehabilitation.

After the successful implementation of the standard work on the inpatient stroke rehabilitation unit, the standard work form was then spread to the two inpatient ABI rehabilitation units at TRI.

\section{RESULTS}

Following the introduction of standard work processes, use of the MTO medical conditions report form increased from $0 \%$ to $100 \%$ over 3 months from February to April 2016. A total of 41 charts were audited. Due to the forcing function, the previous non-standardised form was successfully eliminated from the stroke rehabilitation unit and further data collection of this process measure was deemed not to be required (figure 1). At the end of the project in April 2017, use of the MTO Medical Conditions form continued to be $100 \%$.

A total of 142 charts were audited. At baseline, $50 \%$ of PMR consultations indicated the plan for MTO reporting. After implementation of the standard work form, the proportion of consultations documenting the plan for driving improved. However, with PMR consultant staff changeover, there was a drop in documentation between March and May 2016. A shift was noted starting in September 2016, with MTO reporting properly documented in PMR consultations at or near $100 \%$ for 6 months until February 2017 (figure 2).

One hundred and thirty-two discharge summaries were audited to determine if MTO reporting status was included. Communicating reported drivers in the discharge summary sent to community family physicians improved from $0 \%$ to $90 \%$ in April 2017, with a clear shift towards improvement after July 2016 (figure 3).

Staff interviews revealed that the physicians on the unit were satisfied with the new standard work. There was no significant increase to staff workload, and the process was well integrated into the current workflow.

The hospital received 10 formal patient complaints related to license suspension and lack of information provided regarding the reporting and return to driving process in the 5 years leading up to the project. However,

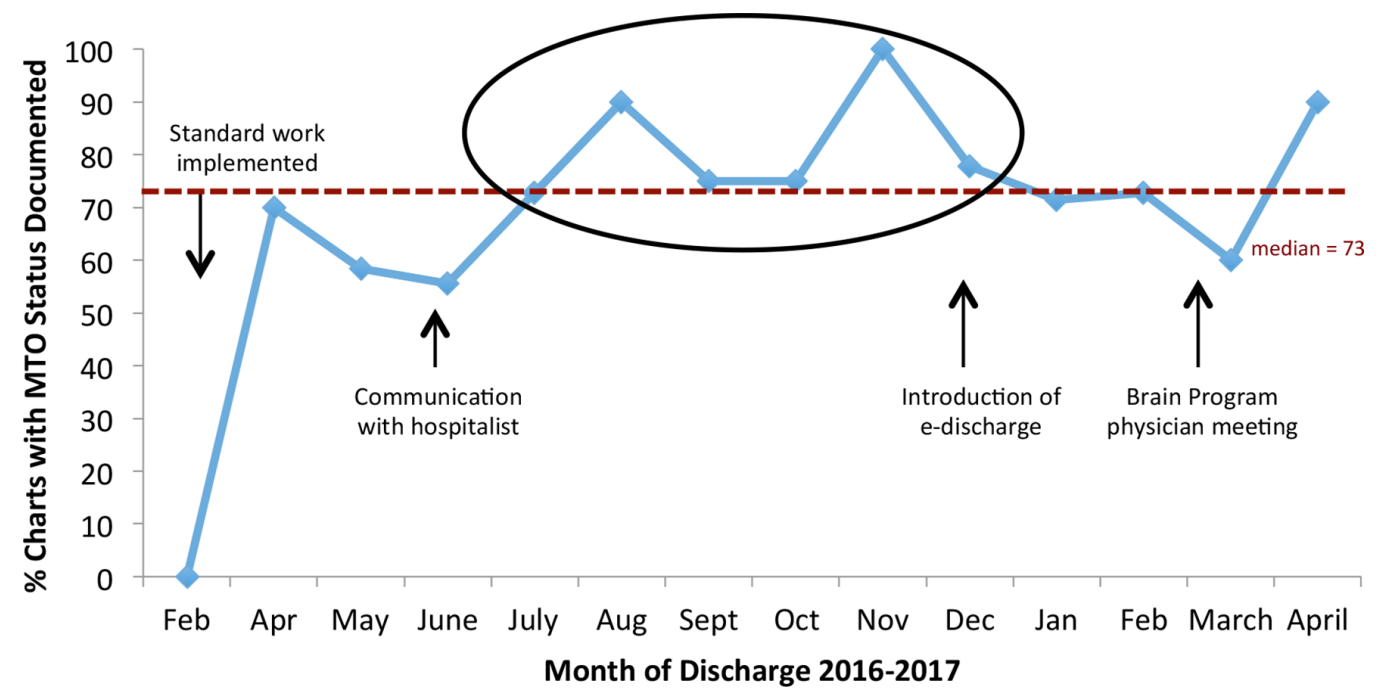

Figure 3 Percentage of discharge summaries documenting patient MTO reporting status. MTO, Ministry of Transportation of Ontario. 
there have been no formal hospital complaints regarding driving in the 14 months since the inception of the project.

\section{LESSONS AND LIMITATIONS}

Despite our education sessions for the physicians on the stroke rehabilitation unit, staff changeover posed a consistent challenge for sustaining our results. Our initial meeting with stakeholders did not include all PMR consultants. We realised the importance of in-person meetings as opposed to email communication to have all stakeholders invested in the change processes.

When we started this endeavour, there were certain factors that were not accounted for, including staff vacations and rotating medical trainees, resulting in coverage by individuals unfamiliar with the initiative. In order to mitigate these, we liaised with PMR staff to ensure that all trainees were familiar with the standard work. We were initially unaware of the e-discharge initiative, which may have been a confounding factor for the discharge summary results.

We did not perform monthly prospective PDSA cycles throughout the entire span of the project, which was a part of the initial strategy. Although this data was eventually collected retrospectively, the delay in data collection may have resulted in missed opportunities to address issues regarding MTO reporting in the PMR consultation in a more timely fashion.

One of the major limitations of this project was that patient satisfaction regarding the reporting process as well as the education provided to them was not evaluated, although the number of patient complaints received was used as a surrogate measure. Our institution plans to formally evaluate patient satisfaction with these processes in the future.

\section{CONCLUSION}

Through the use of Lean methodology, the practice of reporting our stroke rehabilitation inpatients' driver status to the MTO was successfully optimised and standardised. This has improved the communication around driving reporting status with patients and their healthcare providers and decreased the number of patient complaints received regarding this sensitive issue. The improvements in documentation in discharge summaries and PMR consultation notes have been sustained for over 1 year, and as a result, the standard work form is currently being implemented more widely across our hospital, starting with our ABI inpatient units.

Acknowledgements The autors would like to thank the other members of the Toronto Rehabilitation Institute Driving Working Group: Brianna Bourne, Andrea Calvet, Kelly Dostrovsky, Jacqualyn Davies, Hamed Ghotbi, Robert Hastings, Debbie Hebert, Geoff Law, Edith Ng, Chris Pita and Susan Varughese. They would also like to acknowledge Sarah Cote, for administrative support, and the staff on the inpatient stroke rehabilitation unit, in particular the hospitalist, Dr Cary Fan.

Contributors SLM, PLJ and IJC: responsible for conducting interviews, data collection, analysis and had a significant role in drafting the manuscript. SLM: submitted the manuscript. MB: facilitated organisation of LEAN process improvement events, advised SLM, PLJ and IJC in data collection, and guided the manuscript writing and editing of all drafts, including preparing responses to reviewers. AL: planned and supervised the study design and all aspects of the study.

Funding The project was supported by in-kind contributions of staff time from the Toronto Rehabilitation Institute. Mark Bayley receives funding support from the Saunderson Family Chair at the Toronto Rehabilitation Institute Foundation.

Competing interests None declared.

Provenance and peer review Not commissioned; externally peer reviewed.

Open access This is an open access article distributed in accordance with the Creative Commons Attribution Non Commercial (CC BY-NC 4.0) license, which permits others to distribute, remix, adapt, build upon this work non-commercially, and license their derivative works on different terms, provided the original work is properly cited, appropriate credit is given, any changes made indicated, and the use is non-commercial. See: http://creativecommons.org/licenses/by-nc/4.0/.

\section{REFERENCES}

1. Toronto Rehab Institute. UHN. http://www.uhn.ca/TorontoRehab/ About/Pages/default.aspx (cited 16 Jul 2017).

2. Krueger $\mathrm{H}$, Koot J, Hall RE, et al. Prevalence of individuals experiencing the effects of stroke in canada: trends and projections. Stroke 2015;46:2226-31.

3. Public Health Agency of Canada. Tracking heart disease and stroke in Canada - stroke highlights 2011. 2011. http://www.phac-aspc.gc.ca/ cd-mc/cvd-mcv/sh-fs-2011/index-eng.php (cited 1 Jul 2017).

4. CMA Driver's Guide. Determining medical fitness to operate motor vehicles: Canadian Medical Association. 2012. https://www.cma.ca/ En/Pages/drivers-guide.aspx (cited 1 Jul 2017).

5. Perrier MJ, Korner-Bitensky N, Petzold A, et al. The risk of motor vehicle crashes and traffic citations post stroke: a structured review. Top Stroke Rehabil 2010;17:191-6.

6. Frith J. Returning to driving after stroke: a systematic review of adherence to guidelines and legislation. Br J Occup Ther 2015;78:349-55.

7. White $\mathrm{JH}$, Miller $\mathrm{B}$, Magin $\mathrm{P}$, et al. Access and participation in the community: a prospective qualitative study of driving post-stroke. Disabil Rehabil 2012;34:831-8.

8. Finestone HM, Guo M, O'Hara P, et al. Driving and reintegration into the community in patients after stroke. PM\&R 2010;2:497-503. 